\section{CONTRAST ENHANCED MICRO-COMPUTED TOMOGRAPHY RESOLVES THE 3-DIMENSIONAL MORPHOLOGY OF THE CARDIAC CONDUCTION SYSTEM IN MAMMALIAN HEARTS}

doi:10.1136/heartjnl-2012-302951.021

Presenter: Robert Stephenson

${ }^{1} S$ Robert. Stephenson, ${ }^{2} \mathrm{R}$ Mark Boyett, ${ }^{2}$ George Hart, ${ }^{2}$ Theodora Nikolaidou, ${ }^{2}$ Xue Cai, ${ }^{3} \mathrm{~F}$ Antonio Corno, ${ }^{4} \mathrm{Nel}$ son Alphonso, ${ }^{1}$ Nathan Jeffery, ${ }^{1} \mathrm{C}$ Jonathan Jarvis. ${ }^{1}$ Department of Musculoskeletal Biology, Institute of Ageing \& Chronic Disease, University of Liverpool, Sherrington Buildings, Ashton Street, Liverpool, L69 3GE, UK; 'Cardiovascular Research Group, School of Medicine, University of Manchester, Core Technology Facility, 46 Grafton St, Manchester M13 9NT, UK; ' ${ }^{3}$ rince Salman Heart Center, King Fahad Medical City, Riyadh, Kingdom of Saudi Arabia; ${ }^{4}$ Alder Hey Children's NHS Foundation Trust, Liverpool, UK

The general anatomy of the cardiac conduction system (CCS) has been known for 100 years, but its complex irregular 3D geometry is not well understood largely because the specialised tissue cannot be easily distinguished from working myocardium. The best anatomical descriptions come from serial sectioning of preparations taken from appropriate areas of the heart. Low X-ray attenuation has formerly ruled out micro-computed tomography (micro-CT) to resolve topology of soft tissue, but incorporation of high molecular weight molecules enhances differential attenuation and allows visualisation of fine detail. Using an iodine based contrast agent, we obtained exquisite high resolution contrast enhanced micro-CT images of cardiac tissue from rat and rabbit in which the three major subdivisions of the CCS can be differentiated from the surrounding contractile myocardium, and visualised in 3D. The sinoatrial node and the associated ring bundle, the atrioventricular conduction axis (including inferior nodal extension and penetrating bundle), His bundle, bundle branches and Purkinje network can be objectively identified by differential attenuation. Purkinje fibres within the ventricles appear both as structures running on the endocardial surface and free running in the luminal cavity. Controversially, analogous structures are present in the atria, mainly on or near to the endocardial surface. Although the current findings are consistent with existing anatomical representations, the new images offer superior resolution and are the first 3D representations of the CCS within intact mammalian hearts. The method promises to improve the anatomical fidelity of computational models designed to understand complex normal and pathological conduction within the heart.

\section{SEMI-AUTOMATIC OEDEMA QUANTIFICATION FROM DIRECT T2 MAP CARDIAC MRI}

Presenter: Colin Berry

doi:10.1136/heartjnl-2012-302951.022

${ }^{1.2} \mathrm{Hao}$ Gao 'David Carrick, ${ }^{3}$ Kushsairy Kadir, 'Christie McComb, 'John Foster, ${ }^{3} \mathrm{~J}$ ohn Soraghan, ${ }^{2}$ Xiaoyu Luo, ' ${ }^{1}$ Colin Berry. ${ }^{1} B H F$ Cardiovascular Research Centre, University of Glasgow, UK; ${ }^{2}$ School of Mathematics and Statistics, University of Glasgow, UK ; ${ }^{3}$ CeSIP, University of Strathclyde, UK

The jeopardised ischaemic area-at-risk (AAR) is a key prognostic determinant in acute myocardial infarction. Myocardial oedema imaging with T2-weighted cardiac magnetic resonance CMR is validated for imaging the AAR and T2 'mapping' is a new method for AAR imaging with clinical and research potential. We aimed to develop an automated post-processing method that would enable straightforward volumetric quantification of AAR with T2 maps. Our approach retains user input (i.e. clinical judgement) to confirm the presence of oedema on an image which is then subjected to an automated analysis. The new method was tested on 12 acute MI patients who had a CMR within 48 hours of hospital admission. Manual segmentation of the left ventricular wall and oedema were available for comparison. Left ventricular wall boundaries were delineated automatically by variational level set methods followed by automated detection of myocardial oedema by fitting a Gaussian-Gaussian mixture statistical model. The mean perpendicular distances between automatically detected left ventricular boundaries and corresponding manual delineated boundaries were $1.8 \pm 0.2 \mathrm{~mm}$ for endocardial boundaries and $2.3 \pm 0.3 \mathrm{~mm}$ for endocardial boundaries. Dice similarity coefficients for agreement $(0=$ no agreement, $1=$ perfect agreement) between manual delineation and automated segmentation of the left ventricular wall boundaries and oedema regions were $0.85 \pm 0.02$ and $0.74 \pm 0.05$, respectively. Compared to standard manual approaches, the new semi-automated method for estimating myocardial oedema is straightforward and accurate.

\section{3 UNCOVERING THE MOLECULAR MECHANISMS OF PATENT FORAMEN OVALE}

doi:10.1136/heartjnl-2012-302951.023

\section{Presenter: Graeme Elliott}

GC Elliott, R Gurtu, W Newman, C McCollum and T Wang. School of Biomedicine, Faculty of Medical and Human Sciences, The University of Manchester

Patent Foramen Ovale (PFO) is a cardiac developmental defect present in $\sim 25 \%$ of the general population. It is a major risk factor in cryptogenic stroke and migraine. PFO occurs when the Foramen Ovale (FO), a valve-like passageway in the foetal heart allowing blood to bypass the lungs, fails to fuse shut remaining open into adulthood. Evidence suggests $\mathrm{PFO}$ has a genetic predisposition. Genetic variants associated with PFO would serve as biomarkers used for screening high risk individuals. Specific genes that contribute to FO closure are largely unknown. This is attributed to our incomplete understanding of the physiological process of $\mathrm{FO}$ closure. To investigate the molecular mechanisms underlying FO closure, gene expression and histological analysis is being performed using rats as a model. For the first time histological analysis of heart tissue sections from rats aged E20 to P7 have uncovered specific contributions of septum primum and se cundum to FO closure. Results from immunostaining of marker genes including vimentin, SM alphaactin and CD-31 have suggested endothelial-to-mesenchymal transition (EndMT) occurs in this process. Based on the fact that patients who have CADASIL, a genetic stroke syndrome caused by NOTCH3 gene mutations, have a high prevalence $(80 \%)$ of $\mathrm{PFO}$; and that Notch signalling is a critical player in EndMT, immunohistochemical staining of FO regions for genes of the Notch signalling pathway have been carried out. Preliminary results showed specific expression patterns of these genes in $\mathrm{FO}$ region. We conclude that $\mathrm{FO}$ closure is a process of EndMT with the involvement of Notch signalling.

\section{INITIAL RESULTS OF SIMULTANEOUS PET/MRI EVALUATION OF THE INFARCTED MOUSE HEART}

Presenter: Victoria Pell

doi:10.1136/heartjnl-2012-302951.024

${ }^{1} \mathrm{C}$ Methner, ${ }^{2} \mathrm{G}$ Buonincontri, 'VR Pell, ${ }^{2} \mathrm{SJ}$ Sawiak, ${ }^{2} \mathrm{TA}$ Carpenter, and ${ }^{1 T} \mathrm{~T}$ Krieg. ${ }^{1}$ Department of Medicine and; ${ }^{2}$ Wolfson Brain Imaging Centre, University of Cambridge, UK

Background Chronic heart failure (CHF), as a result of acute myocardial infarction (AMI), is currently a leading cause of death worldwide. The direct assessment of novel treatments targeting CHF in vivo however remains difficult. While MRI can reveal cardiac morphology and tissue perfusion through the use of gadolinium (Gd) contrast agents, PET provides information on myocardial function at the molecular level. Here we present preliminary data using a 